\title{
Plattform II „In die Tiefe gehen“
}

Margret Hemme, Projektleiterin Plattform II

Erste Aktion: Bildhauerisches Arbeiten in Kooperation mit Mitgliedern des Kunstvereins Lingen, Kunsthalle, jenseits der Bahn

Zweite Aktion: TeilnehmerInnen: 12 „BildhauerInnen“ und 5 „JournalistInnen“, Kinder der Klassen 7 und 8 der Pestalozzischule Lingen, Förderschule Lernen Ort: am Bahnhof, diesseits des Tunnels, 27. Juni bis 1. Juli 2005, täglich von 10 bis 13 Uhr

In Vorgesprächen mit dem Lehrer Georg Rosen wurde der Termin für das Projekt auf das Ende des Schuljahres gelegt: Kinder und LehrerInnen haben zu diesem Zeitpunkt Tests und Konferenzen hinter sich und können sich besser einlassen. Die Einteilung in BildhauerInnen und JournalistInnen - ursprünglich sollten die BildhauerInnen auch die JournalistInnen sein - erfolgte auf Vorschlag eines weiteren Lehrers, Dr. Lars Göhmann. Er betreut in der Schule die Theatergruppe und wollte zusätzlich mit diesen Kindern teilnehmen. Beide Lehrer nahmen zeitweise am Projekt teil. Erste Informationen erhielten die Teilnehmerlnnen durch einen Brief und durch die beiden Lehrer, die in drei vorausgegangenen Treffen mit dem Projekt vertraut gemacht wurden.

\section{SKIZZE EINER WOCHE ${ }^{1}$}

„Wie man es erzählen kann, so ist es nicht gewesen.“ Christa Wolf

Montag: Kunstschule: Töpferraum, zwischen Sand, Steinen und Werkzeugen, zwischen Kakao-, Kaffee- und Brötchenduft frühstücken 15 Kinder, zwei Lehrer und unsere Crew. Gespannte Erwartung auf allen Seiten. Die fremde Umgebung macht die Schülerlnnen verlegen und neugierig. Der erste Hunger ist gestillt. Wir erzählen von unserer Arbeit. Fragen tauchen auf: WAS MACHT IHR HIER? MACHT IHR KUNST? WAS IST KUNST? KANN NUR EIN KÜNSTLER KUNST HERSTELLEN?

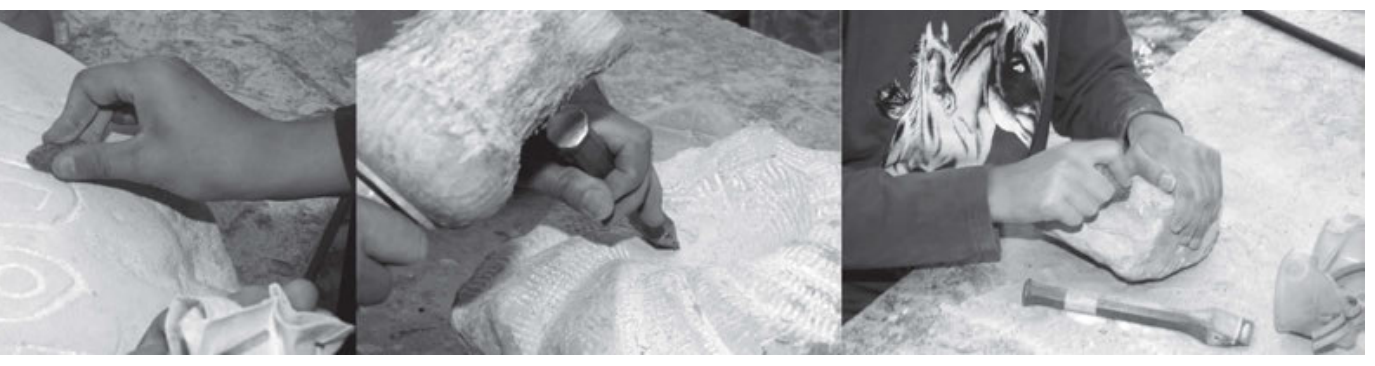


Endlich kommen wir zum „Tunnel“: WIE SOLL ER AUSSEHEN? WAS SIND TUNNELSTEINE? Gedanken bewegen sich auf Neuland. Am Bahnhof, neben dem kubikmetergroßen, leuchtend gelben Sandsteinhaufen, unter dem Dach des Pavillons, Erfahrungen mit Stein und Werkzeug und mit den eigenen Möglichkeiten. Eine ältere Frau, die ich als Teilnehmerin von der ersten Aktion wiedererkenne, wartet schon. Die Gedanken der Schülerlnnen sind förmlich zu spüren: WAS WILL DIE OMA DENN HIER? Ihre kleine Figur, die sie auspackt, ruft Bewunderung hervor: IST DAS SCHWIERIG? SO WAS MÖCHTE ICH AUCH KÖNNEN! Steine ausSUchen: GANZ SCHÖN SCHWER DIESE BROCKEN; WER HILFT MIR TRAGEN? DER IST MIR ZU DICK, ICH NEHME EINEN ANDEREN! WIE MUSS ICH DEN MEISSEL HALTEN UND DEN KNÜPFEL? Der Himmel ist bewölkt. WIE SOLL MEIN TUNNELSTEIN AUSSEHEN? NICHT MIT DEN STEINEN WERFEN! ICH SCHLAGE EIN HERZ; EIN TUNNELHERZ, LIEBE IM TUNNEL. Lachen. Kratzgeräusche, der Meißel hinterlässt Spuren, breite oder schmale, die Knüpfel treffen das Eisen - nicht immer - das Klopfen wird rhythmischer, der feine Sand fasziniert, stört, er fliegt im Wind. Hinter den Schutzbrillen wird es warm, ja heiß, PassantInnen bleiben stehen: WAS MACHT IHR HIER? HIER SOLL EIN TUNNEL GEBAUT WERDEN, WIR BEARBEITEN TUNNELSTEINE!

Flugs ist es 12.30 Uhr, aufräumen, es hat nicht geregnet.

Die JournalistInnen überlegen: WAS WOLLEN WIR FRAGEN, WIE SPRECHEN WIR MENSCHEN AN, WEN FRAGEN WIR? Sie erstellen einen Fragebogen.

Dienstag: Die Bildhauerlnnen trudeln ein, der Pavillon wird aufgebaut, das Werkzeug muss geholt werden. LOS LEUTE, MITHELFEN, NICHT NUR DIE MÄDCHEN! Das Klopfen beginnt, die Spuren von gestern werden vertieft, der Stein verändert sich. Einige sind noch völlig unschlüssig. Gestalterische Freiheit stellt Anforderungen. Andere entwickeln konkrete Formvorstellungen, das Werkzeug wird ausprobiert. IHR KÖNNT AUCH DIE BOHRMASCHINE BENUTZEN. Alle haben einen Bohrmaschinenführerschein. Mit dem Bohrhammer ist es nicht so einfach. Sie trauen sich. Es „schockt“, den Stein mit massiver Kraft zu bearbeiten. Die ersten Brüche. WAS NUN? Da kommt Hilfe, ein Teilnehmer der Sonntagsaktion, gelernter Bildhauer, zeigt einige Kniffe, flink skizziert er seine Ideen auf den Stein.

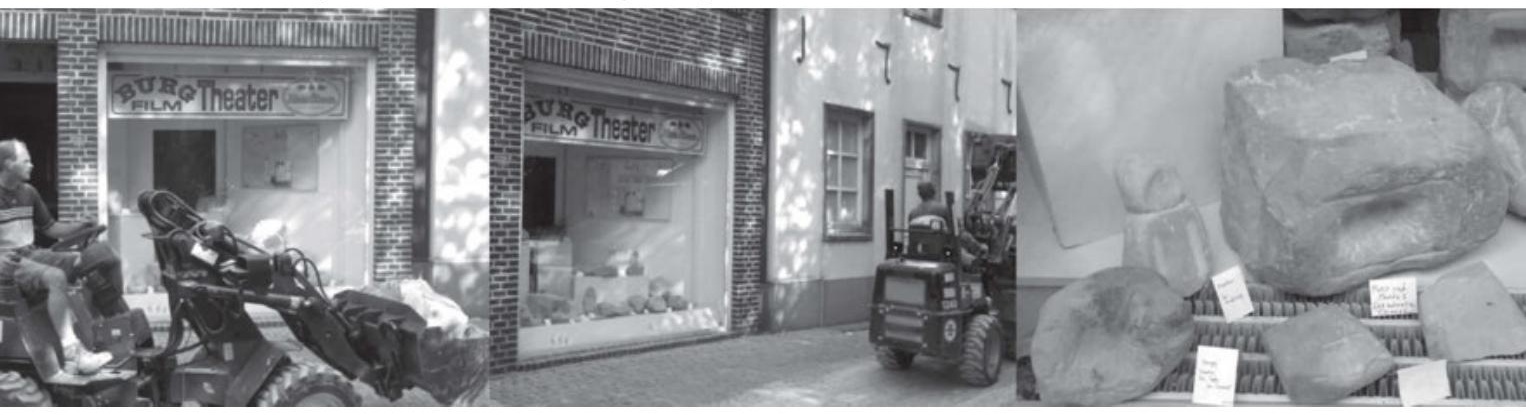

Plattform II 
HALT! An dieser Stelle greife ich ein, wobei mir wichtig ist: Technische Hilfe ja, auch Ideenfindung im Gespräch, aber keine Übernahme vorgefertigter Ideen. Zu viele Details überfordern die Kids, die Ausführung gelingt nicht, Vereinfachung ist angesagt. Steine zeigen schon Formen, sie regen Vorstellungen an. Phantasie und Wahrnehmung sind gefragt. Tamara entdeckt in ihrem Stein eine Figur, andere sehen Linien, Höhen, Tiefen. Andrea hat etwas vor, sie redet nicht. Annikas bildhauerische Ansätze schlängeln über den Stein. Jasmin verfolgt ihre Herzidee, ein Schatten spielt in der Herzvertiefung. Die Arbeit ist anstrengend. ICH KANN NICHT MEHR. WIR HABEN HUNGER! Frühstücken, laufen, rennen, die Gegend erkunden. Wir müssen sie suchen. Eine ältere Dame aus der Nachbarschaft staunt über die Ausdauer der Jugendlichen, sie begrüßt den Tunnelbau, für sie ein bequemer Weg nach drüben. Gegen Mittag hat einer seinen Stein zu Sand verarbeitet, SANDSTEIN. WIE ENTSTEHEN STEINE? AUS DEM SAND IM MEER, DURCH DRUCK, WELCHE STEINE SIND HÄRTER? MARMOR ODER GRANIT? NEE, GRANIT. Steingespräche, einer will sich im Internet erkundigen.

Die JournalistInnen kommen vorbei, berichten über ihre Interviews, sie fotografieren.

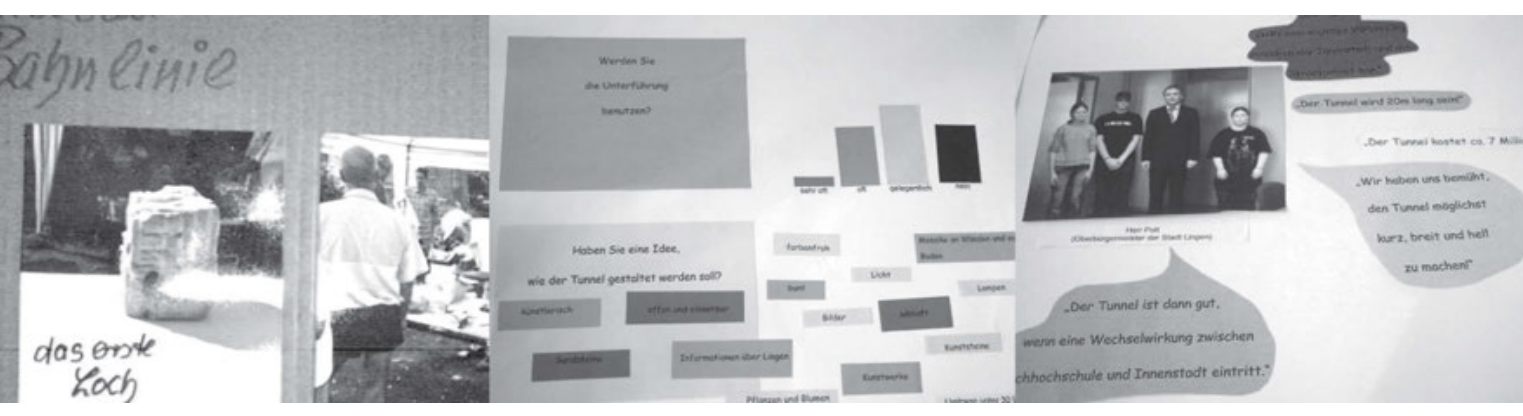

Mittwoch: Es ist trocken, der „Sandmann“ wuchtet einen neuen Stein zum Tisch. Er beginnt, vorsichtiger jetzt, die Erfahrenen geben Ratschläge, viele gehen ganz gezielt an die Arbeit. Ruhiges, regelmäßiges: Poch, Kling, Poch, Poch, Krach. SCHEISSE, DER FUSS, WO IST DER FUSS? WEG! MIST! WAS MACH ICH NUN? Ratschläge von mehreren Seiten, ein neuer Stein muss her, oder doch nicht? DIE FORM KÖNNTE ICH ÄNDERN, DIE LINIEN ANDERS VERLAUFEN LASSEN. Die Figur wird weiter bearbeitet; der Zufall kreativ genutzt. Maik hat in den zwei vorangegangenen Tagen einen Fisch „gefangen“; wir schauen uns das Flachrelief eines Lachses aus prähistorischer Zeit an (Höhlenmalerei, M. Lorblanchet), ihm fällt auf, dass sein Fisch nicht auf dem Stein liegt, er hat ihn hineingearbeitet, ein versenktes Relief. Er mag diese Arbeit mit dem Stein. Andrea setzt ihr Schweigen beharrlich fort, sie hat die Ritztechnik für sich entdeckt. Der „Sandmann“ reduziert wieder das Volumen. Der Sand rieselt, der Staub liegt auf den Gesichtern, in und 
auf der Kleidung, die alten Kittel kommen nun doch zum Zuge. Hin und wieder büchsen einige aus. Beliebt ist das Schleifen mit dem Schmirgelstein, vier glatte, handschmeichelnde Flächen entwickeln sich zu einer „Pyramide“, was glatt ist wird noch glatter, immer wieder fühlen, herrlich. HATTEN WIR HEUTE SCHON 'NE PAUSE? Gleich ist Mittag, die halbe Kiste Wasser ist leer. Der „Sandmannstein“ gestaltet sich zum Sofa. Titel: „WARTEN IM TUNNEL“. Aufräumen ist unbeliebt.

Die JournalistInnen haben inzwischen, ganz alleine ohne Lehrer (darauf haben sie bestanden), den Bürgermeister interviewt.

Donnerstag: Letzter voller Arbeitstag. Bedauern? Freude? Die Ferien winken.

Die ersten fertigen Steine werden präsentiert. PassantInnen fragen immer wieder nach dem Tun, die SchülerInnen informieren sie gerne. Heute ist die Presse da. Frau Tondera stellt Fragen und fotografiert. KOMMEN WIR IN DIE ZEITUNG? Zwei Schüler wollen auf keinen Fall fotografiert werden. Inzwischen ist klar, dass neben Tunneleingängen und Durchbrüchen auch eine Pyramide, ein Hund, ein Herz und das Sofa im vorläufig imaginären Tunnel Platz finden müssen, die „Tunnelphantasie“ ist warm gelaufen. Fragen nach dem Verbleib der fertigen Steine evozieren Lösungsvorschläge: WIR KÖNNTEN IN DER SCHULE EINE MAUER BAUEN, NEIN! DIE STEINE SOLLEN IM TUNNEL EINGEBAUT WERDEN. Die Initiatoren werden sich darum bemühen. Heute staubt es besonders stark. Zwischen Klopfen und Betrachten im abgeschlagenen Sand wühlen, Spuren ziehen, das fasziniert. Sand, Sand, herrlich gelber Sand rieselt, verweht, krabbelt auf der Haut, in der Nase, knirscht zwischen den Zähnen, die „BildhauerInnen“ gleichen den Steinen. Marco und Markus bearbeiten zusammen einen Stein. Der Sandsteinhaufen schrumpft.

Ich bin gespannt auf unseren letzten Tag.

Die JournalistInnenen haben ihre Interviews statistisch ausgewertet, sie versuchen eine Präsentation ihrer Erfahrungen.

Freitag: Es ist trocken. Die Idee, einen Stein für die Leiterin der Tanzschule anzufertigen, die unsere Werkzeuge Tag für Tag beherbergt hat, nehmen zwei Mädchen auf. Sie machen Vorschläge für die Gestaltung und nehmen die Ausführung in die Hand. Letztes Klopfen, Schmirgeln und Kratzen. Überraschungsfrühstück, gestiftet von einer Lehrerin, die Kids genießen es. Großes Aufräumen. Um 12 Uhr ist der Platz leer. Die Tunnelsteine sind in der Kunstschule ausgestellt, mit Titeln versehen. Die Ergebnisse der Interviews sehen wir auf Schautafeln. MitschülerInnen, die Schulleiterin, weitere Lehrerlnnen sind gekommen, werden nach einer Begrüßung durch die Kunstschulleitung von den Journalistlnnen und den BildhauerInnen über ihr Tun in dieser Woche informiert. Die Vernissage ist eröffnet. Fragen zum Tunnelbau, zu den Interviews und zu den Erfahrungen mit Steinen, Staub, Werkzeugen prasseln auf die AkteurInnen ein, die viel erzählen, fachgerecht, infor- 
miert und stolz. Nicht nur die Steine haben sich in dieser Woche verändert.

Die Lehrer überreichen jedem/r „Bauarbeiterln“ eine Urkunde.

REFLEXION: 22 Stunden haben ca. 40 BauarbeiterInnen (15 SchülerInnen und ca. 25 Erwachsene) auf der Baustelle Kunst gearbeitet, den Blick auf den Tunnel für sich und andere erweitert, sie sind mit Fragen, Bohrer, Hammer und Meißel in die Tiefe gegangen. Dadurch sind Veränderungen eingetreten, nicht nur an den Steinen.

\section{Anmerkungen}

1 Die Grundlage für die folgende Rekonstruktion bildet ein Gedächtnisprotokoll, das ich nach jedem Projekttag anfertigte.

- Die JournalistInnen mussten sich über den Tunnelbau informieren, ein Konzept entwickeln, welche Informationen sollen wie und an wen weitergegeben, wie das Erfahrene dargestellt werden.

- BürgerInnen der Stadt wurden über den Tunnelbau informiert, ihre Meinung war gefragt, zur Gestaltung und Nutzung des Tunnels.

- Die BildhauerInnen haben sich mit Werkzeug und Material vertraut gemacht, zur Gestaltung Ideen entwickelt, die Steine umgewandelt nach eigenen Vorstellungen.

- BildhauerInnen und JournalistInnen haben die Prăsentation ihrer Werke geplant, ihre Ausstellung wurde von zwei weiteren Klassen der Pestalozzischule besucht.

- Die Stadtwerke Lingen begrüßten die Aktion, sie stellen Öberlegungen an, wie die angefertigten Steine im Tunnel eingebaut werden können. Lingen wird steinreich!

- Fragen und Diskussionen ergaben sich aus den Berührungspunkten mit PassantInnen, Bürgermeister, Tunnelbau, Projekt, Kunstschule ...

- Ich, als Plattforminitiatorin, erfuhr Neues über den Tunnelbau, über „BauarbeiterInnen“ und Beton; immer wieder musste ich meine pädagogischen Schritte anders setzen, um keinem auf die Fuße zu treten, davonzueilen oder hinterherzuhinken. Mut zum eigenen Ausdruck, Ausdauer und Zielstrebigkeit wurden bewiesen. Es entwickelte sich Freude an den eigenen kreativen Făhigkeiten, Neugier auf den Verlauf und das Ergebnis der gemeinsamen Aktion (nicht nur bei den InitiatorInnen). Es gab auch zeitweise Verweigerung der Mitarbeit einiger SchülerInnen, mangelndes Zutrauen der Erwachsenen in die Kreativität der SchülerInnen, Stress bei SchülerInnen und Erwachsenen. Die Arbeitsatmosphäre habe ich insgesamt als motivierend beurteilt. Wir haben voneinander und miteinander gelernt. Wir haben einen Prozess in Gang gesetzt, der eine Richtung hatte, der Wege anbot, deren Verlauf und Qualităt von den TeilnehmerInnen selber gestaltet wurden.

Alle diese Wege zusammen ergeben einen Ort: einen Ort, der wie der Tunnel ein Durchgang sein kőnnte. In diesem Fall ein Durchgang in Richtung Kunst. 


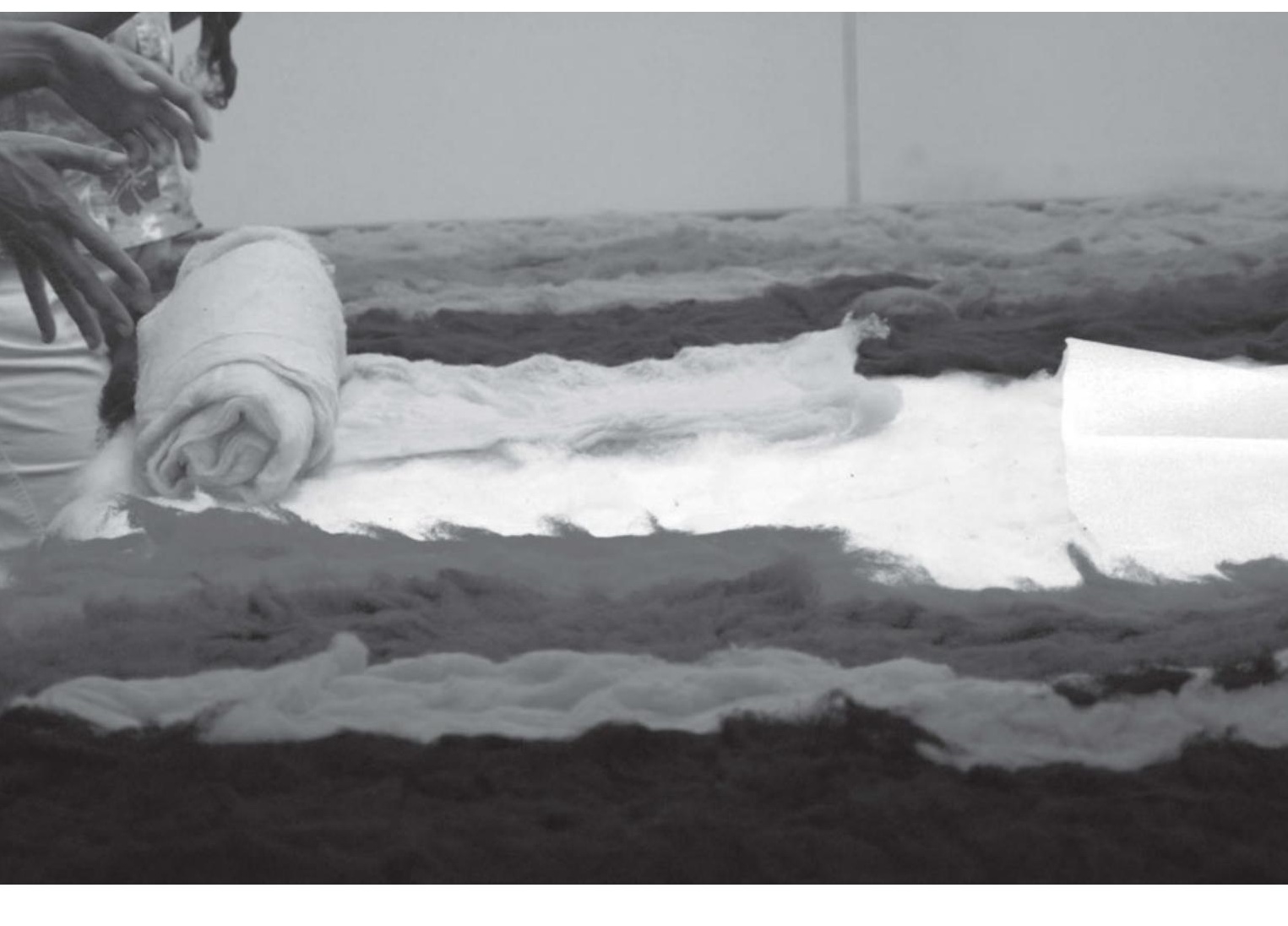

